\title{
Searches for Sterile Neutrinos with NOvA
}

\section{G. S. Davies*}

Indiana University

E-mail: gsdaviesdiu.edu

\section{A. Aurisano, A. Sousa, S. Yang}

University of Cincinnati

E-mail: aurisaameuc.edu, alex.sousa@uc.edu, yangs9@uc.edu

\section{G. K. Kafka}

Harvard University

E-mail: gkafka@fas.harvard.ed

\section{S. Kasetti}

University of Hyderabad, India

Email: kasettisivaprasadegmail.com

\section{R. Keloth}

Cochin University of Science and Technology, India

Email: rijeeshkodencheri@gmail.com

\section{Suter}

Fermi National Accelerator Laboratory

Email: lsuterefnal.gov

Contradictory evidence has been presented on the issue of neutrino mixing between the three known active neutrinos and light sterile neutrino species. Apparent short-baseline neutrino oscillations observed by the LSND [1] and MiniBooNE [2] experiments, the collective evidence of the reactor neutrino anomaly, and the gallium anomaly all point towards sterile neutrinos with mass at the $1 \mathrm{eV}$ level. While these results are tantalizing, they are not conclusive as they are in tension with null results from other short-baseline experiments, and with disappearance searches in longbaseline and atmospheric experiments. The NOvA (NuMI Off-Axis $v_{e}$ Appearance) experiment may help clarify the situation by searching for disappearance of active neutrinos from the NuMI (Neutrinos from the Main Injector) beam over a baseline of $810 \mathrm{~km}$. We describe the method used by NOvA to look for oscillations into sterile neutrinos at the Far Detector (FD) through the disappearance of neutral-current (NC) neutrino events, including preliminary results of this search. In addition, the Near Detector (ND) is well suited for searching for anomalous short-baseline oscillations and probing the LSND and MiniBooNE sterile neutrino allowed regions using a variety of final states. We also present a novel method for selecting samples with high purity at the ND using convolutional neural networks. Based on this method, the sensitivity to anomalous short-baseline $v_{\tau}$ appearance are shown, and searches for anomalous $v_{e}$ appearance and $v_{\mu}$ disappearance at the NOvA ND are presented.

38th International Conference on High Energy Physics

3-10 August 2016

Chicago, USA

${ }^{*}$ Speaker. 


\section{Introduction}

The three neutrino paradigm has been used to successfully explain the vast majority of neutrino oscillation results, but there are a few results that can not be explained without the introduction of a additional neutrinos. The LSND [1] and MiniBooNE [2] short-baseline experiments saw an excess of $\bar{v}_{e}$ appearance which can be interpreted as oscillations with a light neutrino with a mass on order of $1 \mathrm{eV}$. High precision measurements of the decay width of the $\mathrm{Z}$ boson performed by four LEP experiments [3] showed that only three light active neutrino flavors exist. It is therefore concluded that any additional neutrinos must be sterile, as in they must not interact through the weak force.

NOvA is an off-axis long-baseline neutrino experiment. The ND measures the neutrino energy spectrum before three-flavor oscillations, predicting the FD spectrum with corrections for acceptance and flux differences derived from simulation). The ND is $1 \mathrm{~km}$ from the target at Fermilab, $105 \mathrm{~m}$ underground. The FD is located on the surface in Ash River, Minnesota. Both detectors are sited $14.6 \mathrm{mrad}$ off the central axis of the beam, observing neutrinos predominantly in a narrow range of energies between 1 and $3 \mathrm{GeV}$ in order to match to the first 3-flavor oscillation maximum for the NOvA baseline. The NOvA detectors are functionally equivalent tracking calorimeters, composed of cells of liquid scintillator encased in polyvinyl chloride (PVC) extrusions. By looking for a deficit of neutral-current events (NC) in the three-flavor oscillation formalism, NOvA can search for light sterile neutrinos at the FD. The NC disappearance analysis in the FD presents a model-independent interpretation of the results using data from a $v_{\mu}$ beam and a baseline of 810 $\mathrm{km}$. The NOvA ND will be sensitive to oscillations due to a $1 \mathrm{eV}$ scale sterile neutrino. This note also briefly describes the NOvA potential for the short-baseline oscillations using a joint fit between $v_{\mu}$ disappearance and $v_{e}$ appearance and a search for $v_{\tau}$ appearance.

\section{NC Disappearance Analysis}

Neutral-current interactions are not affected by three-flavor oscillations but mixing between active and sterile neutrinos will reduce the rate of $\mathrm{NC}$ events at the FD, as sterile neutrinos will not interact in the detector volume. This analysis takes advantage of recent advances in machine learning and tools from the computer vision community to classify neutrino interactions. The Convolutional Visual Network (CVN) [4] event selection algorithm is based on the GoogLeNet convolutional neutral network $(\mathrm{CNN})$ architecture. Figure 1 shows the CVN NC classifier distribution for the FD-selected data compared to the three-flavor prediction for NC events, beam and cosmogenic backgrounds after preselection and cosmic background rejection cuts have been applied. The CVN neutral current event classifier is the primary selector for the signal selection and achieves excellent efficiency (94\%) and purity (88\%) when separating the NC signal from beam backgrounds; $50 \%$ efficiency and $72 \%$ purity when cosmic backgrounds are included.

NOvA observes $95 \mathrm{NC}$ candidate events between $0.5-4 \mathrm{GeV}$ in the FD after performing a cutand-count analysis to obtain a single value result. Given the extrapolated prediction of $83.71 \pm$ 9.15 (stat.) \pm 8.28 (syst.), the observation in data is within one sigma of three-flavour prediction. NOvA sees no evidence for sterile neutrino mixing with the $6.05 \times 10^{20}$ POT of neutrino data analysed to date. The calorimetric energy distribution of events selected in the FD is show in Fig. 2 compared to the 3 -flavour signal and background simulated prediction. 

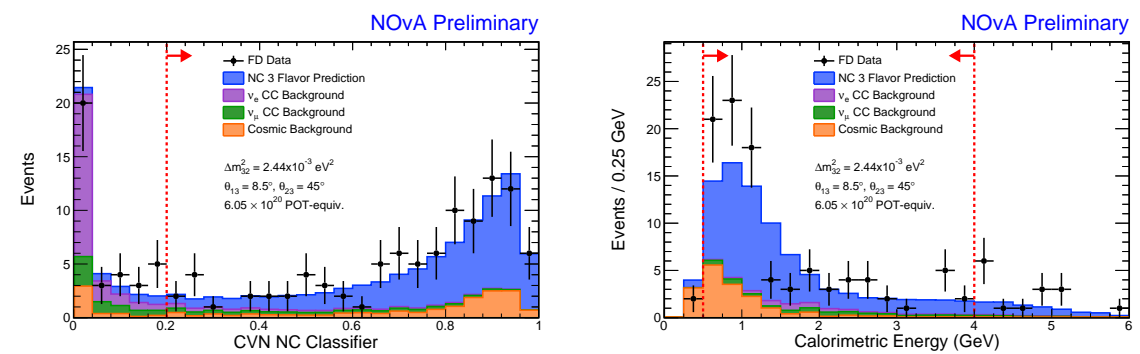

Figure 1: The CVN NC event classifier. Figure 2: The distribution of events seRed dashed line and arrow depict the se- lected at the NOvA FD. Red dashed lines lection region above the CVN NC classi- and arrows depict the calorimetric energy fier value of 0.2 for this analysis. range 0.5-4.0 GeV selection region.

An R-ratio can be constructed to check consistency with the standard 3-flavour prediction. The R-ratio is a ratio of the predicted background from all neutrino flavors and cosmic ray muons $\left(B_{C C+\text { cosmic }}\right)$ subtracted from the number of data-selected NC events $\left(N_{\text {Data }}\right)$ and the predicted NC signal $\left(S_{N C}\right)$. For clarity $\mathrm{R}$ is:

$$
R=\frac{N_{\text {Data }}-\Sigma B_{C C+\text { cosmic }}}{S_{N C}}
$$

This analysis calculates $R=1.19 \pm 0.16(\text { stat } .)_{-0.13}^{+0.08}($ syst. $)$. An R-ratio comparison with the standard 3-flavor model concludes that the NC disappearance result is consistent with 3-flavour oscilations $(R=1.0)$. Further details of the NC disappearance analysis can be found here [5].

\section{Short-Baseline Oscillations}

If a light sterile neutrino exists, one can expect to observe disappearance in muon neutrinos and appearance in electron neutrinos at the NOvA ND, due to oscillations with mass squared difference between the active and sterile neutrino, $\Delta m_{41}^{2}>1 e V^{2}$. In the $3+1$ model, $v_{e}$ appearance can be expressed as:

$$
\begin{aligned}
P_{v_{\mu} \rightarrow v_{e}}^{S B L}= & 4\left|U_{\mu 4}\right|^{2}\left|U_{e 4}\right|^{2} \sin ^{2} \frac{\Delta m_{41}^{2} L}{4 E} \\
& =\sin ^{2} 2 \theta_{\mu e} \sin ^{2} \frac{\Delta m_{41}^{2} L}{4 E}
\end{aligned}
$$

where $\sin ^{2} 2 \theta_{\mu e}=\sin ^{2} 2 \theta_{14} \sin ^{2} \theta_{24}$ and $v_{\mu}$ disappearance can be expressed as:

$$
\begin{gathered}
P_{v_{\mu} \rightarrow v_{\mu}}^{S B L}=1-4\left|U_{\mu 4}\right|^{2}\left(1-\left|U_{\mu 4}\right|^{2}\right) \sin ^{2} \frac{\Delta m_{41}^{2} L}{4 E} \\
=1-\sin ^{2} 2 \theta_{\mu \mu} \sin ^{2} \frac{\Delta m_{41}^{2} L}{4 E}
\end{gathered}
$$

where $\sin ^{2} 2 \theta_{\mu \mu}=\cos ^{2} \theta_{14} \sin ^{2} \theta_{24}$ [6]. Since both $v_{e}$ appearance and $v_{\mu}$ disappearance probabilities are parameterized in $\theta_{14}$ and $\theta_{24}$, minimizing the total $\chi^{2}$ from both of them simultaneously with respect to $\theta_{14}$ and $\theta_{24}$ gives the potential to constrain both of these angles. The NOvA sensitivity at $90 \%$ C.L for short-baseline oscillations due to light sterile neutrinos is shown in Fig. 3 for one and three years of data taking. NOvA's one year 90\% C.L sensitivity excludes LSND 90\% and 99\% C.L results at $\Delta \mathrm{m}^{2}>1 \mathrm{eV}^{2}$ region and is in tension at $\Delta \mathrm{m}^{2}<1 \mathrm{eV}^{2}$. The three year 90\% C.L 
sensitivity completely excludes the LSND $90 \%$ and $99 \%$ C.L regions. NOvA can probe the LSND and MiniBooNE allowed regions with one NOvA TDR-equivalent year of data. The NOva ND can also probe for $v_{\tau}$ appearance. Figure 4 shows the $2 \mathrm{D}$ exclusion region with a rate-only fit assuming a two-flavour approximation to the $3+1$ model at short-baselines. NOvA will be competitive with previous experiments after 3 years of running in this channel.
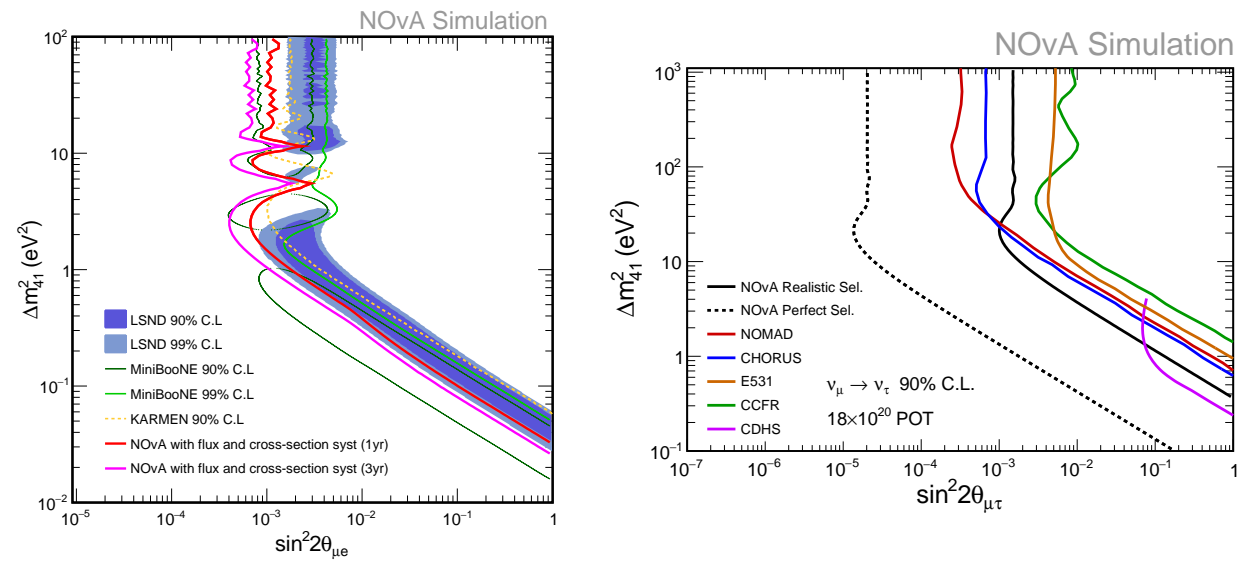

Figure 4: $90 \%$ C.L sensitivity for $\Delta m_{41}^{2}$ Figure 3: Sensitivity for $\theta_{\mu e}$ at $90 \%$ C.L.; and $\sin ^{2} 2 \theta_{\mu \tau}$ after 3 years. The regions profiling over $\theta_{34}$ at $90 \%$ C.L. The re- excluded by CDHS, CCFR, E531, NOgions excluded by LSND, MiniBooNE MAD and CHORUS are also shown. and KARMEN are also shown.

\section{Acknowledgements}

This work was supported by the U.S. Department of Energy; the U.S. National Science Foundation; the Department of Science and Technology, India; the European Research Council; the MSMT CR, Czech Republic; the RAS, RMES, and RFBR, Russia; CNPq and FAPEG, Brazil; and the State and University of Minnesota. We are grateful for the contributions of the staffs at the University of Minnesota module assembly facility and Ash River Laboratory, at the Argonne National Laboratory, and at Fermilab. Fermilab is operated by Fermi Research Alliance, LLC, under Contract No. De-AC02-07CH11359 with the U.S. DOE.

\section{References}

[1] A. Aguilar et al. [LSND Collaboration], Phys. Rev. D 64 (2001) 112007

[2] A. A. Aguilar-Arevalo et al. [MiniBooNE Collaboration], Phys. Rev. Lett. 110 (2013) 161801

[3] S. Schael et al., Phys. Rept. 427 (2006) 257-454

[4] A. Aurisano, A. Radovic, D. Rocco et al., JINST 11 (2016) P09001

[5] G. S. Davies, http://theory.fnal.gov/jetp/talks/jetp_gsdavies_nova-2.pdf (2016)

[6] J. Kopp et al., JHEP 1305 (2013) 050 